\title{
Sparse Localization with a Mobile Beacon Based on LU Decomposition in Wireless Sensor Networks
}

\author{
Chunhui ZHAO, Yunlong XU, Hui HUANG \\ College of Information and Communication Engineering, Harbin Engineering University, Harbin 150001, P. R. China \\ zhaochunhui@hrbeu.edu.cn, xuyunlong@ hrbeu.edu.cn, huanghui@hrbeu.edu.cn
}

\begin{abstract}
Node localization is the core in wireless sensor network. It can be solved by powerful beacons, which are equipped with global positioning system devices to know their location information. In this article, we present a novel sparse localization approach with a mobile beacon based on LU decomposition. Our scheme firstly translates node localization problem into a 1-sparse vector recovery problem by establishing sparse localization model. Then, $L U$ decomposition pre-processing is adopted to solve the problem that measurement matrix does not meet the restricted isometry property. Later, the 1-sparse vector can be exactly recovered by compressive sensing. Finally, as the 1-sparse vector is approximate sparse, weighted Centroid scheme is introduced to accurately locate the node. Simulation and analysis show that our scheme has better localization performance and lower requirement for the mobile beacon than $M A P+G C, M A P-M$, and $M A P-M \& N$ schemes. In addition, the obstacles and DOI have little effect on the novel scheme, and it has great localization performance under low SNR, thus, the scheme proposed is robust.
\end{abstract}

\section{Keywords}

Wireless sensor network, mobile beacon, localization, LU decomposition, compressive sensing

\section{Introduction}

With the development of hardware and wireless technology, wireless sensor network (WSN) is broadly used in many applications, such as disaster relief, environmental observation, military surveillance, and so on. WSN is composed by a large number of low-cost and energy-limited sensor nodes, which are randomly deployed in the sensing region. Various operations in WSN such as the network geographic coverage, deployment and localization of sensor nodes are worthy of consideration. Clearly, sensor location information plays a key role for all these issues, besides that, it is also helpful for detecting where specific events happen, tracking mobile target, promoting traffic routing, and so on. Therefore, the techniques to ensure the location of sensor nodes are of critical importance in WSN.
Due to the constraints of cost and power consumption, it is impractical to equip all sensor nodes with global positioning system (GPS) devices. However, it is feasible to equip GPS for few nodes called beacons, in practical applications. Beacons can be static or mobile, which can usually be used to locate other nodes in WSN. The existing localization techniques can be classified into range-based schemes and range-free schemes. The range-based schemes need to measure the distance between two neighbor nodes [1-6], in which the distance is calculated based on received signal strength (RSS), time of arrival (TOA), time difference of arrival (TDOA), or angle of arrival (AOA). The range-based schemes have two obvious weaknesses: the nodes require additional measuring equipment and the localization accuracy is vulnerable to the environmental interference. Many range-free schemes have been proposed so far [7-14], in which, each node utilizes the information obtained from the few beacons to estimate its location. However, the node must be in the sensing range of beacons. Of course, the information that a node cannot directly communicate with a beacon can also be used to ensure the tighter border on its location.

Although beacons are powerful, they cost a lot in WSN. To minimize the number of beacons and complete localization for other nodes, a beacon which can move and periodically broadcast its location to its one-hop neighboring nodes is used to replace dense stationary beacons [15-22]. These approaches have shown the localization based on one mobile beacon or few mobile beacons is more accurate and cost-effective than the localization based on stationary beacons.

In WSN, it is a hot research field to apply compressive sensing (CS) [23], [24] to target localization [25-27]. Unlike the Nyquist theorem, CS just needs few noisy measurements to reconstruct the signal, which is sparse or compressible under a transform basis. It can recover exactly the original sparse signal with high probability by solving a minimization problem [28-30]. In the novel target localization algorithm based on CS theory [25], a preprocessing is introduced to make the measurement matrix meet the restricted isometry property (RIP) [24], so the performance can be guaranteed. Zhao and $\mathrm{Xu}$ [26] proposed a novel multiple target localization algorithm based on Bayesian CS. It can effectively reduce the energy consumption, but it may bring about false targets. Zhang et al. [27] presented 
a greedy matching pursuit (GMP) algorithm to reconstruct the signal. Compared with traditional algorithms, GMP has better anti-noise performance and localization performance.

In this article, we apply CS to node localization and present a novel node localization scheme with a mobile beacon in WSN - sparse localization approach with a mobile beacon based on LU decomposition (SLMLU). This scheme can accurately localize nodes as the sensor node is unique in its sensing area. SLMLU firstly builds a sparse localization model by utilizing the mobile beacon information (including the location and RSS sent by mobile beacon at each beacon point) collected by sensor node. Secondly, it introduces LU decomposition preprocessing to ensure that measurement matrix meets RIP. Then, SLMLU recovers the sparse signal exactly by CS. Finally, it gets the location of each sensor node by using weighted centroid scheme to solve the problem that the sparse signal is not exact 1 -sparse vector.

The major contributions of this article are as follows:

1) We propose the SLMLU algorithm which can effectively improve the localization performance compared with other algorithms.

2) We present a sparse localization model by the uniqueness of the sensor node in its sensing area, and this model can translate node localization problem into CS problem.

3) We utilize LU decomposition preprocessing to make the measurement matrix meet RIP.

The rest of the paper is organized as follows. Section 2 concerns the related works on localization in WSN. In Sec. 3, we propose a novel model for node localization. Section 4 describes the detail of SLMLU. The performance of SLMLU, MAP+GC, MAP-M, and MAP-M\&N schemes is in Sec. 5. Finally, Section 6 relates to the conclusion.

\section{Related Works}

To solve localization problems in WSN, many schemes have been proposed in many literatures. Excellent surveys of the related studies can be found in [31-32]. The existing localization techniques are summarized briefly in this section.

\subsection{Range-based Schemes}

The range-based schemes are used to estimate locations by measuring either distances or angles. There are lots of approaches, such as strategies based on RSS, TOA, TDOA, and $\mathrm{AOA}$, to calculate the distance between two neighbor nodes in range-based schemes. For example, Bahl et al. [1] converted the received signal strength to distance information and made use of the triangulation to estimate a node's location. Bergamo and Mazzini [2] proposed a triangulation strategy for localization and analyzed the effects of fading and sensor mobility. Priyantha et al. [3] used the beacon advertisements received by node to distance measuring and then estimated its possible locations. Niculescu and Nath [4], Nasipuri and Li [5] proposed some other schemes to estimate relative angles between one-hop neighboring nodes for angulations. Brida et al. [6] propose an enhanced DV-AOA algorithm. The angular information of nodes is ensured and processed by the original algorithm; however, the final location estimation is determined by the proposed novel algorithm using only a subset of all intersections.

\subsection{Range-free Schemes}

The range-free schemes are more cost-effective than the range-based schemes, as they are without limitation of the hardware device. Brida et al. [7] give a survey of two proximity based location techniques performance, which is not the most accurate techniques, but a low cost alternative to more expensive techniques. Belusu et al. [8] proposed centroid scheme in which centriod formula was adopted to determine node's locations based on received beacons' locations. Jun et al. [9] and Yang et al. [10] improved centroid scheme by RSS technology. Niculescu and Nath [11] introduced DV-Hop scheme to estimate the location of sensor nodes by computing hop counts from each node to specific beacons. In [12], the DV-Hop scheme was improved by RSS technology. Bulusu et al. [13] proposed an approximate point-in-triangulation test (APIT) scheme. It firstly selects three one-hop neighboring nodes of a node, then, confirms whether or not the node is in the triangle ensured by the three beacons, and finally, estimates the location of the node. Tran and Nguyen [14] presented a localization scheme in wireless sensor networks based on support vector machines (LSVM), in which they introduce support vector machine (SVM) to identify the location of each node.

\subsection{Mobile Beacon}

The authors proposed many schemes by using one mobile beacon or few mobile beacons, these schemes can also be divided into range-based and range-free.

Sichitiu and Ramadurai [15] presented a range-based localization algorithm. They estimate locations of nodes by using RSS technique to deal with the beacon messages heard from single mobile beacon. In [16], the authors proposed a probabilistic localization method based on a mobile beacon. It comprises TOA technique and Centroid formula to range and compute locations of nodes. Xia and Chen [17] proposed a localization scheme, which determines locations of nodes by using TDOA technique and trilateration.

Xiao et al. [18] proposed a range-free localization scheme - half arrival and departure overlap (HADO). The scheme uses arrival and departure constraint area to decide the location of each node. However, HADO has a strict 
requirement for the path of mobile beacons. In [19], the authors proposed a range-free localization scheme. It uses directional antennas to estimate each node location, but needs to equip mobile beacon with four directional antennas. Ssu et al. [20] proposed a localization scheme with mobile anchor points (MAP). It uses the information received from the mobile beacon to find two chords of a circle, and lets the center of the circle be the location of senor node. On this basis, Lee et al. [21] presented $\mathrm{MAP}+\mathrm{GC}$ scheme. MAP $+\mathrm{GC}$ is a geometric constraint algorithm, in which the intersection area is obtained by two reference points. Liao et al. [22] proposed two improved MAP algorithms called MAP-M and MAP-M\&N. These algorithms obtain two possible locations when the centers and radii of two circles are known, and then ensure the location of node according to beacon or neighbor node.

\section{Sparse Localization Model}

CS offers a novel framework to reconstruct the sparse signal or compressible signal in a certain basis, with far fewer measurements than the traditional techniques. It explores an $l_{1}$-minimization problem to recover the sparse signal. To make this possible, two basic elements must be guaranteed in CS: sparsity (the signal must be sparse or compressible) and incoherence (the measurement matrix must meet RIP).

As the sensor node is unique in the sensing area, it can be modeled as an ideal 1-sparse vector. Divide the sensing area into $N$ grids (see Fig. 1), and then the location of the sensor node over the grid is described by $\boldsymbol{X}$ as follows:

$$
\boldsymbol{X}=\left(x_{1}, \cdots, x_{n}, \cdots, x_{N}\right)^{T}
$$

where $\boldsymbol{X}$ is a $N$ by 1 column vector, $(\cdot)^{T}$ means transpose, $x_{n}(1 \leq n \leq N, N$ is the number of grid) is a element of $\boldsymbol{X}$, which describes the $n$-th grid. If the sensor node lies in the $i$-th grid, $x_{i}$ equals to 1 and the other elements of $\boldsymbol{X}$ are 0 . Thus, the node localization problem can be well formulated as a 1-sparse vector recovery problem in sparse localization model, as shown in Fig. 1.

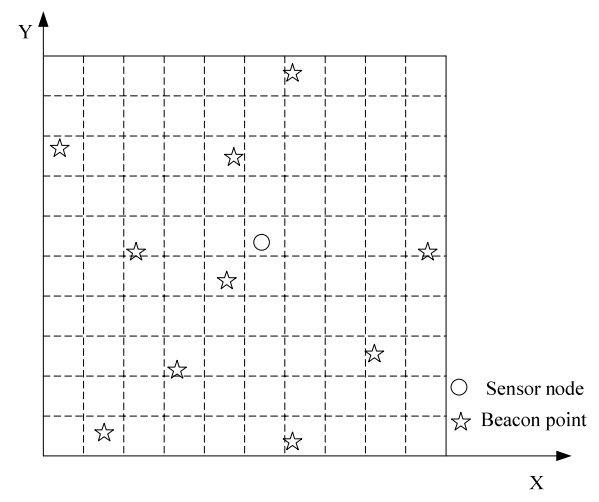

Fig. 1. Sparse localization model.

In the sparse localization model, $R_{m, n}$ represents the RSS between the $m$-th beacon point and the center location of the $n$-th grid, which is the element of measurement matrix $\boldsymbol{A}$. Thus, CS process of the sparse signal $\boldsymbol{X}$ can be described as:

$$
\boldsymbol{Y}=\left[\begin{array}{c}
y_{1} \\
y_{2} \\
\vdots \\
y_{M}
\end{array}\right]=\left[\begin{array}{cccc}
R_{1,1} & R_{1,2} & \cdots & R_{1, N} \\
R_{2,1} & R_{2,2} & \cdots & R_{2, N} \\
\vdots & \vdots & \ddots & \vdots \\
R_{M, 1} & R_{M, 2} & \cdots & R_{M, N}
\end{array}\right]\left[\begin{array}{c}
x_{1} \\
x_{2} \\
\vdots \\
x_{N}
\end{array}\right]=\boldsymbol{A} \boldsymbol{X}
$$

where $\boldsymbol{Y}$ is a $M$ by 1 measurement vector, $y_{m}$ is the RSS from the $m$-th beacon point to the sensor node. If the sensor node lies in the $i$-th grid, $x_{i}$ is equal to 1 , otherwise $x_{i}$ is equal to 0 . Obviously, the signal $\boldsymbol{X}$ is a 1 -sparse signal.

\section{The Proposed Scheme}

\subsection{LU Decomposition Pre-processing}

As the measurement matrix $\boldsymbol{A}$ is composed of the RSS between $M$ beacon points and all grids, it cannot satisfy RIP. In order to solve this problem, SLMLU introduces a data pre-processing for measurement matrix $\boldsymbol{A}$ and measurement vector $\boldsymbol{Y}$. The process is as follows.

Theorem 1 (LU Decomposition) [33]: If $\boldsymbol{A} \in \boldsymbol{C}_{M}^{M \times N}$, $\boldsymbol{A}$ can be only decomposed by the following formula:

$$
\boldsymbol{A}=\boldsymbol{L} \boldsymbol{U}
$$

where $\boldsymbol{U}$ and $\boldsymbol{L}$ are $M$ by $N$ unitary matrix and $M$ by $M$ lower triangular matrix, respectively, $\boldsymbol{U}$ can be ensured by Schmidt orthogonalization and normalization [33], and $\boldsymbol{U} \boldsymbol{U}^{T}=\boldsymbol{I}_{M}$.

According to Theorem 1, it can be found that the measurement matrix $\boldsymbol{A}$ can be decomposed into a unitary matrix $\boldsymbol{U}$ and a lower triangular matrix $\boldsymbol{L}$ by LU decomposition, and $\boldsymbol{U}$ can be obtained by Schmidt orthogonalization and normalization.

The Schmidt orthogonalization process can be given by

$$
\boldsymbol{B}_{m}=\boldsymbol{A}_{m}-\frac{\left\langle\boldsymbol{A}_{m}, \boldsymbol{B}_{1}\right\rangle}{\left\langle\boldsymbol{B}_{1}, \boldsymbol{B}_{1}\right\rangle} \boldsymbol{B}_{1}-\cdots-\frac{\left\langle\boldsymbol{A}_{m}, \boldsymbol{B}_{m-1}\right\rangle}{\left\langle\boldsymbol{B}_{m-1}, \boldsymbol{B}_{m-1}\right\rangle} \boldsymbol{B}_{m-1}
$$

where $m=1, \ldots, M,\langle\cdot, \cdot\rangle$ expresses the dot-product of vectors, $\boldsymbol{A}_{m}$ and $\boldsymbol{B}_{m}$ are the $m$-th row vectors of $\boldsymbol{A}$ and $\boldsymbol{B}$, respectively. The row vectors of $\boldsymbol{B}$ are orthogonal to each other.

Then, $\boldsymbol{U}$ can be ensured by the following Schmidt normalization process

$$
\boldsymbol{U}=\left[\begin{array}{cccc}
1 /\left\|\boldsymbol{B}_{1}\right\| & 0 & \cdots & 0 \\
0 & 1 /\left\|\boldsymbol{B}_{2}\right\| & \cdots & 0 \\
\vdots & \vdots & \ddots & \vdots \\
0 & 0 & \cdots & 1 /\left\|\boldsymbol{B}_{M}\right\|
\end{array}\right] \boldsymbol{B}
$$


where $\left\|B_{i}\right\|$ is the modulus of the vector $B_{i}$, which is $l_{2}$ norm of the vector $B_{i}$. Finally, a new measurement matrix $\boldsymbol{Q}$ can be obtained by renormalizing each column of the unitary matrix $\boldsymbol{U}$

$$
\boldsymbol{U}=\boldsymbol{Q}\left[\begin{array}{ccccc}
\| \boldsymbol{U}_{1} & 0 & \cdots & 0 \\
0 & \| \boldsymbol{U}_{2} & \cdots & 0 \\
\vdots & \vdots & \ddots & \vdots \\
0 & 0 & \cdots & \left\|\boldsymbol{U}_{N}\right\|
\end{array}\right]
$$

where $\boldsymbol{U}_{1}, \boldsymbol{U}_{2}, \ldots, \boldsymbol{U}_{N}$ are column vectors of the unitary matrix $\boldsymbol{U}$. From the above analysis and [23], it can be seen that the measurement matrix $\boldsymbol{Q}$ is an incoherent measurement (one of the most common measurement matrices in CS). Therefore, the measurement matrix $\boldsymbol{Q}$ can completely meet RIP.

The same procedure is carried out to obtain a new measurement vector $\boldsymbol{Y}^{\prime}$, which is expressed as follows:

$$
\begin{aligned}
\boldsymbol{Y}^{\prime} & =(\boldsymbol{L})^{*} \boldsymbol{Y}=(\boldsymbol{L})^{*} \boldsymbol{A} \boldsymbol{X}=(\boldsymbol{L})^{*} \boldsymbol{L} \boldsymbol{U} \boldsymbol{X} \\
& =\boldsymbol{U} \boldsymbol{X}=\boldsymbol{Q}\left[\begin{array}{cccc}
\| \boldsymbol{U}_{1} & 0 & \cdots & 0 \\
0 & \| \boldsymbol{U}_{2} & \cdots & 0 \\
\vdots & \vdots & \ddots & \vdots \\
0 & 0 & \cdots & \left\|\boldsymbol{U}_{N}\right\|
\end{array}\right] \boldsymbol{X}=\boldsymbol{Q} \boldsymbol{X}^{\prime}
\end{aligned}
$$

where $(\cdot)^{*}$ is the inverse of a matrix. As $\boldsymbol{U} \boldsymbol{U}^{T}=\boldsymbol{I}_{M}$, $\boldsymbol{L}=\boldsymbol{A} \boldsymbol{U}^{T}$. Via the above formula, $\boldsymbol{X}^{\prime}$ can be represented as

$$
\boldsymbol{X}^{\prime}=\left[\begin{array}{cccc}
\| \boldsymbol{U}_{1} & 0 & \cdots & 0 \\
0 & \| \boldsymbol{U}_{2} & \cdots & 0 \\
\vdots & \vdots & \ddots & \vdots \\
0 & 0 & \cdots & \left\|\boldsymbol{U}_{N}\right\|
\end{array}\right] \boldsymbol{X}
$$

Since $\boldsymbol{X}$ is sparse, $\boldsymbol{X}^{\prime}$ is ensured to multiply $\boldsymbol{X}$ by a diagonal matrix, thus, $\boldsymbol{X}^{\prime}$ also is sparse.

\subsection{RIP Analysis}

Definition 1 [24]. A matrix $\boldsymbol{\Phi}$ obeys RIP with parameters $(k, \delta)$ for $\delta \in(0,1)$ if

$$
1-\delta \leq \frac{\|\boldsymbol{\Phi} x\|_{2}^{2}}{\|x\|_{2}^{2}} \leq 1+\delta
$$

holds for all $k$-sparse vector $x$.

In [23], the most common measurement matrices, which can obey RIP, include Gaussian measurements, binary measurements, Fourier measurements, and incoherent measurements. The definition of incoherent measurements is shown as follows.

Definition 2 [23]. Incoherent measurement $\boldsymbol{\Phi}$ is obtained by selecting $M$ rows uniformly at random from an $N$ by $N$ orthonormal matrix $\boldsymbol{U}_{N}$ and renormalizing the columns.

From the above definition, the incoherent measurement $\boldsymbol{\Phi}$ is ensured by renormalizing the columns of an $M$ by $N$ partial orthonormal matrix. What's more, $\boldsymbol{U}$ obtained by LU decomposition is a $M$ by $N$ unitary matrix, in other words, $\boldsymbol{U}$ is also a partial orthonormal matrix. Therefore, $\boldsymbol{Q}$ is an incoherent measurement, which is obtained by renormalizing the columns for $\boldsymbol{U}$, that is, $\boldsymbol{Q}$ can completely meet RIP.

Above all, $\boldsymbol{X}^{\prime}$ is sparse, and $\boldsymbol{Q}$ can completely meet RIP, so $\boldsymbol{X}^{\prime}$ can be accurately recovered by the new measurement vector $\boldsymbol{Y}^{\prime}$, thus $\boldsymbol{X}$ can be ensured.

\subsection{Location Estimation}

As the sensor node does not often lie in the center of the grid, the recovered signal $\boldsymbol{X}$ is not an exact 1-sparse vector, but a sparse vector with a large coefficient and a few non-zero small coefficients. Then, a post-processing procedure is conducted by using weighted centroid algorithm [13-15] to estimate the location. The process is as follows.

First, normalization for the recovered signal $\boldsymbol{X}$ is made as follows:

$$
\omega_{n}=x_{n} / \sum_{n=1}^{N} x_{n}
$$

where $\omega_{n}$ is the weighted coefficient of the center location of the $n$-th grid. Then, the location of the sensor node $(x, y)$ is ensured as:

$$
(x, y)=\sum_{n=1}^{N} \omega_{n} \cdot\left(x_{n}, y_{n}\right)
$$

where $(x, y)$ is the estimated location of the sensor node, $\left(x_{n}, y_{n}\right)$ is the center location of the $n$-th grid.

\subsection{Sensing Area}

Since the sensor node does not know its position, it is unable to determine the sensing area only by itself. Then the node can ensure the sensing area by the beacon points within its sensing area.

SLMLU scheme determines the sensing area by improving beacon box of Monte Carlo Localization boxed (MCB) algorithm [34]. The beacon box of MCB can be given by

$$
\begin{array}{ll}
x_{\min }=\max _{i=1}^{M}\left(x_{i}-r\right) & x_{\max }=\min _{i=1}^{M}\left(x_{i}+r\right) \\
y_{\text {min }}=\max _{i=1}^{M}\left(y_{i}-r\right) & y_{\text {max }}=\min _{i=1}^{M}\left(y_{i}+r\right)
\end{array}
$$

where $x_{\min }, x_{\max }, y_{\min }$, and $y_{\max }$ are the minimum and maximum values of $x$ and $y$ coordinate of the beacon box, 
respectively. $x_{i}$ and $y_{i}$ are $x$ and $y$ coordinate of the $i$-th beacon point, respectively. $r$ is communication radii of node and $M$ is the number of beacon points.

However, the beacon box described above cannot make sure all beacon points within the box. Therefore, SLMLU improves the beacon box by the following formula:

$$
\begin{array}{ll}
x_{\min }^{\prime}=\min \left\{x_{i}, \max _{i=1}^{M}\left(x_{i}-r\right)\right\} & x_{\max }^{\prime}=\max \left\{x_{i}, \min _{i=1}^{M}\left(x_{i}+r\right)\right\} \\
y_{\text {min }}^{\prime}=\min \left\{y_{i}, \max _{i=1}^{M}\left(y_{i}-r\right)\right\} & y_{\text {max }}^{\prime}=\max \left\{y_{i}, \min _{i=1}^{M}\left(y_{i}+r\right)\right\}
\end{array}
$$

where $x_{\min }^{\prime}, x_{\max }^{\prime}, y_{\text {min }}^{\prime}$, and $y_{\text {max }}^{\prime}$ are the minimum and maximum values of $x$ and $y$ coordinate of sensing area, respectively.

\subsection{The Number of Beacon Points}

In [23], the study shows the sparse signal can be exactly recovered by CS theory, while the number of beacon points $M$ is larger than $O\left(C \cdot K \cdot \mu^{2} \cdot(\log N)^{4}\right)$, where $C$ is a constant, $\mu=\sqrt{N} \max _{i, j}\left|U_{i, j}\right|$. While $M$ is large, it will increase computation amount. Therefore, in real-world networks, it is very necessary to select $M$ reasonably.

To make a quantitative analysis of $M$, the node localization could be abstracted into target localization in a sensing area. Suppose there are $M$ sensors randomly distributed in a $20 \mathrm{~m} \times 20 \mathrm{~m}$ sensing area, which is divided into a $15 \times 15$ grid, and $S N R=20 \mathrm{~dB}$. As shown in Fig. 2, the relationship between localization performance and $M$ is obtained by running the simulation 100 times for a fixed $M$.

Note that the average location error is decreased with the increase of $M$. The error is about $0.5 \mathrm{~m}$ while $M \geq 4$, what's more, the average location error is almost no change, while $M \geq 8$.

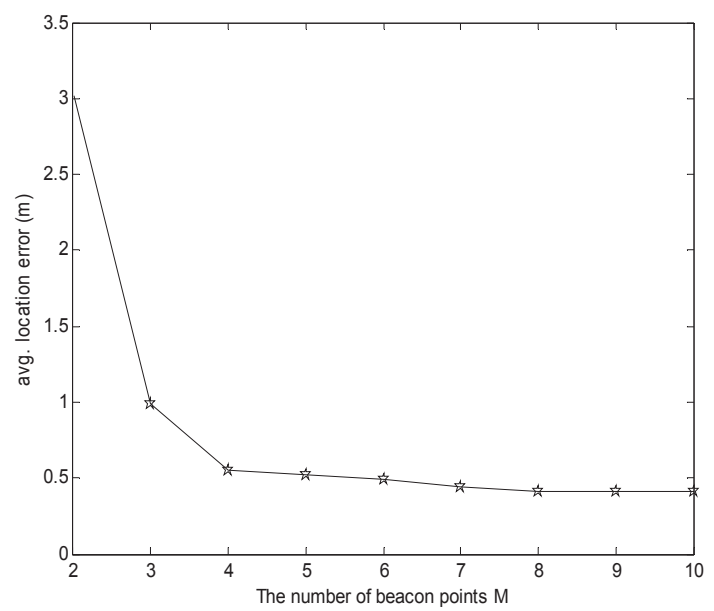

Fig. 2. Relationship between localization performance and $M$.

\section{Simulation Study and Analysis}

The performance of MAP $+\mathrm{GC}$ [21], MAP-M [22], MAP-M\&N [22] and the proposed SLMLU scheme is discussed in this section.

The simulation study is based on a network with 1000 sensor nodes, which are randomly distributed in a $100 \mathrm{~m} \times 100 \mathrm{~m} \mathrm{2D}$ area. Assume the mobile beacon and all the sensor nodes have the same communication range $r=20 \mathrm{~m}$, the mobile beacon is moving straightly with a velocity $20 \mathrm{~m} / \mathrm{s}$, and the random waypoint (RWP) model [35] for the mobile beacon is adopted. The other parameters are as follows: the mobile beacon in $\mathrm{MAP}+\mathrm{GC}$, MAP-M and MAP-M\&N broadcasts a message every $0.1 \mathrm{sec}$ and 10000 times, the chord threshold is specified as $0.3 r$. The beacon distance in $\mathrm{MAP}+\mathrm{GC}$ is $2 \mathrm{~m}$. In the proposed SLMLU, the mobile beacon broadcasts a message every $1 \mathrm{sec}$ and 400 times. The sensing area is divided into a $15 \times 15$ grid. To balance the accuracy and complexity, $M$ equals to the number of beacon points actually received while the number is no more than 8 , otherwise $M$ equals to 8. The signal transmission model employs IEEE 802.15.4 standard [36],

$$
R S S(d)= \begin{cases}P_{t}-40.2-20 \log d, & d \leq 8 \\ P_{t}-58.5-33 \log d, & d>8\end{cases}
$$

where $R S S(d)$ is the strength of the signal received, $P_{t}$ is the strength of the signal transmitted, $d$ is the actual distance of these two nodes.

In this section, we discuss the performance of four schemes under ideal environment (see Fig. 3a) and obstacle environment (see Fig. 3b), and analyzes the effects of radio range, GPS error and the radio irregularity on the performance of four algorithms. It also discusses noise to impact on SLMLU and the computation complexity of four algorithms at the end of the section.

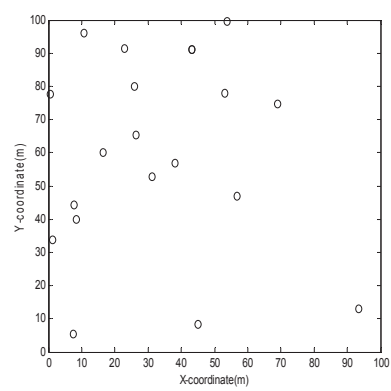

(a)

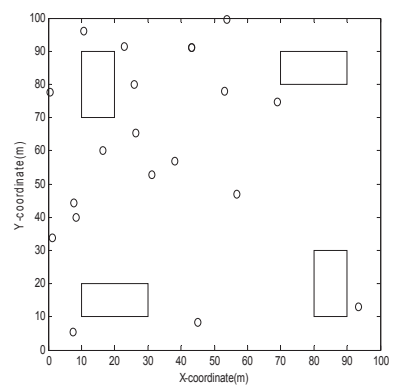

(b)
Fig. 3. Simulation environment: (a) Ideal environment; (b) Obstacle environment.

\subsection{Ideal Environment}

Figure 4 shows location error of each node in ideal environment (see Fig. 3a). 
The circles in Fig. 4(b), Fig. 4(c), and Fig. 4(d) represent the nodes which are not located. As roughly shown in Fig. 4, SLMLU scheme has better localization performance

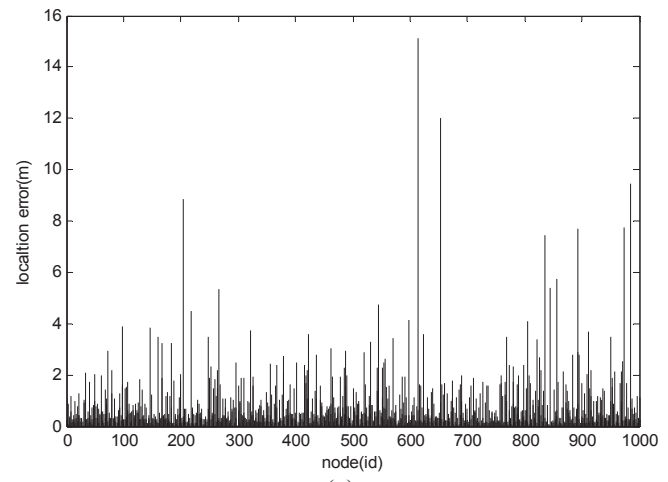

(a)

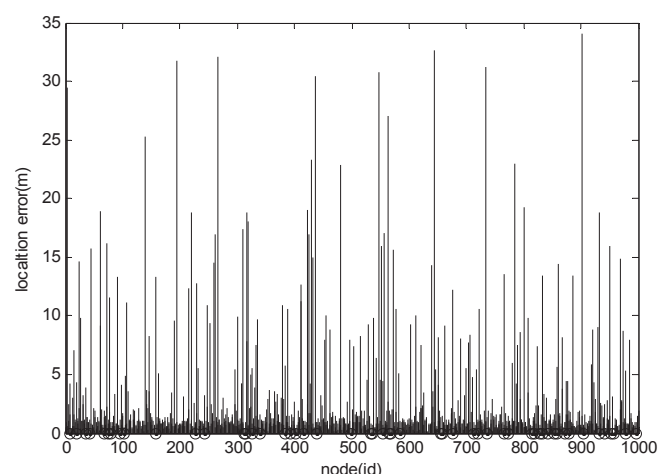

(b)

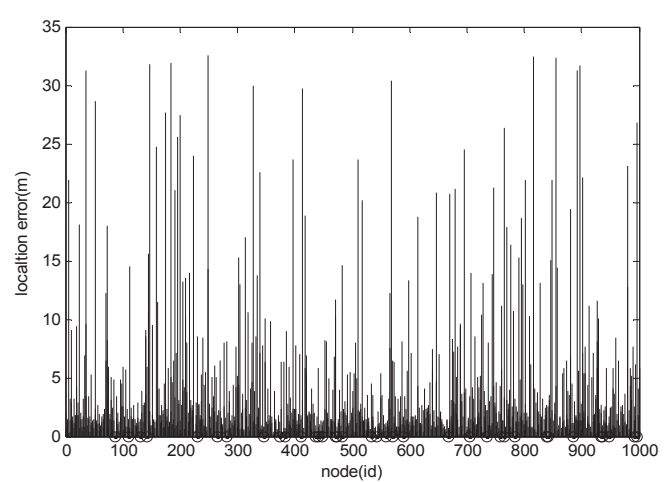

(c)

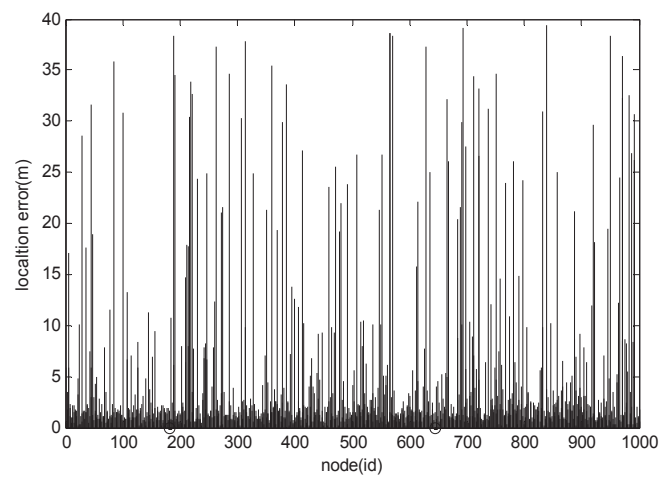

(d)

Fig. 4. The location error of each sensor node. (a) SLMLU; (b) MAP+GC; (c) MAP-M; (d) MAP-M\&N. than MAP+GC, MAP-M and MAP-M\&N schemes. In addition, the location error of SLMLU is more concentrated than $\mathrm{MAP}+\mathrm{GC}, \mathrm{MAP}-\mathrm{M}$ or MAP-M\&N schemes, in other words, SLMLU has smallest location error standard deviation among these four schemes.

It can be also found that the maximum location error of SLMLU is smallest. This is because, in GAP+GC, GAP-M, and GAP-M\&N schemes, the sensor node selects improper beacon points which causes a large location error, but the problem is nonexistent in SLMLU. Besides, although the mobile beacon broadcasts 10000 times, some sensor nodes still cannot be located in MAP+GC, MAP-M and MAP-M\&N. Among these three schemes, MAP+GC scheme has restricted condition, $\mathrm{MAP}+\mathrm{GC}$ has better localization performance than the other two schemes. Comparing with MAP-M, MAP-M\&N has worse localization performance. That is because the located nodes which are used to determine the locations of other nodes in MAP-M\&N may have error, so other nodes may select wrong location.

Table 1 illustrates the average location error of the four algorithms obtained by running Monte Carlo experiment 10 times. Note that the average location error and location error standard deviation of SLMLU scheme is much smaller than that of the other three schemes. Therefore, SLMLU scheme is relatively a good choice for localization.

\begin{tabular}{|c|c|c|}
\hline Scheme & $\begin{array}{c}\text { Avg. location } \\
\text { error }(\mathrm{m})\end{array}$ & $\begin{array}{c}\text { Location error } \\
\text { std. dev. }(\mathrm{m})\end{array}$ \\
\hline SLMLU & 0.73 & 1.48 \\
MAP+GC & 2.06 & 4.45 \\
MAP-M & 2.37 & 4.51 \\
MAP-M\&N & 4.15 & 9.01 \\
\hline
\end{tabular}

Tab. 1. Comparison of localization performance for four schemes.

\subsection{Obstacle Environment}

Obstacles in the sensing area cause radio irregularity in the sensor network. Both the size of the sensing area and the number of sensor nodes are the same as the previous environment. In addition, there are four $10 \mathrm{~m} \times 20 \mathrm{~m}$ obstacles in the area (see Fig. 3b). To gain the robustness of SLMLU scheme, simulation is performed by using the obstacle mobility (OM) model [37]. The OM model assumes that signals can only be received in the line-of-sight (LOS) propagation. Table 2 illustrates the localization performance comparison under obstacle environment for the four methods. As shown, SLMLU has the smallest average location error and location error standard deviation among these four schemes. That is, SLMLU scheme has better localization performance compared with other three schemes in obstacle environment.

According to Tab. 1 and Tab. 2, compared with ideal environment, the four schemes all have worse localization performances under obstacle environment. However, SLMLU proposed has lesser environment impact than 
MAP + GC, MAP-M or MAP-M\&N. Due to existing obstacles, the choice of beacon points is more prone to errors in $\mathrm{MAP}+\mathrm{GC}, \mathrm{MAP}-\mathrm{M}$ and MAP-M\&N schemes, therefore, these three schemes have much worse localization performance. However, SLMLU scheme use RSS to compute the location of the node, so obstacles have little effect on SLMLU scheme.

\begin{tabular}{|c|c|c|}
\hline Scheme & $\begin{array}{c}\text { Avg. location } \\
\text { error }(\mathrm{m})\end{array}$ & $\begin{array}{c}\text { Location error } \\
\text { std. dev. }(\mathrm{m})\end{array}$ \\
\hline SLMLU & 0.91 & 1.81 \\
MAP+GC & 6.26 & 10.51 \\
MAP-M & 6.96 & 10.48 \\
MAP-M\&N & 13.06 & 14.53 \\
\hline
\end{tabular}

Tab. 2. Comparison of localization performance for four schemes.

\subsection{Impact of GPS Error}

All GPS receivers have localization error in real environments, which include three basic location errors (i.e., single-point positioning accuracy, differential positioning accuracy, carrier positioning accuracy [38]).

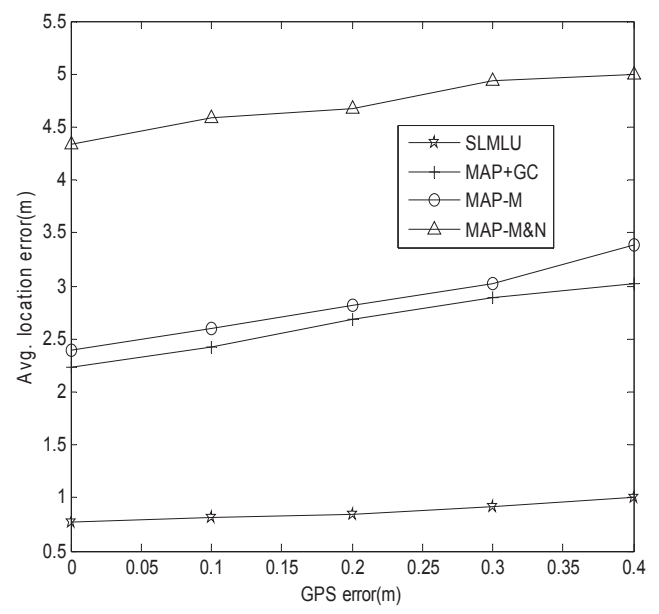

(a)

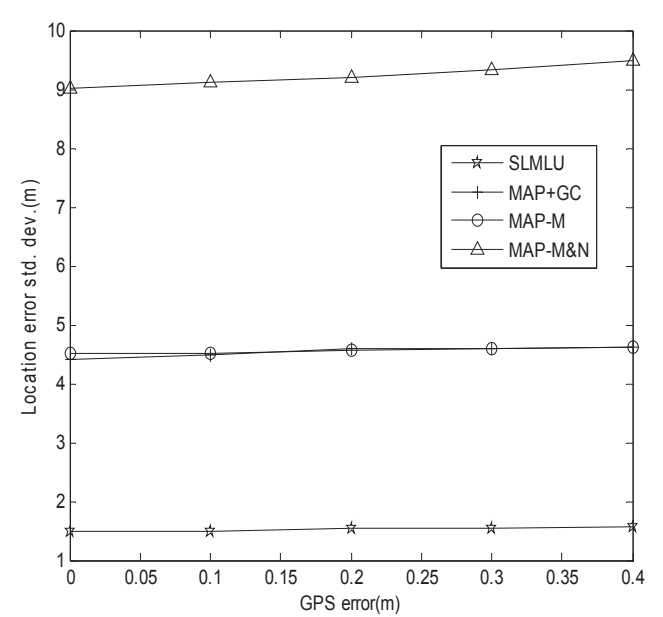

(b)

Fig. 5. Relationship between localization performance and GPS error. (a) Average location error. (b) Location error standard deviation.
The simulations apply the carrier positioning error, assume GPS errors based on a normal distribution, and specify the mean GPS errors as $0,0.1,0.2,0.3$ and 0.4 meter, respectively, with a standard deviation of 0.05 meters [19], [21].

Figure 5 shows the variation of the average location error and location error standard deviation with the GPS error for the four methods. In general, with the increase of the GPS errors, the average location errors and location error standard deviations of the four methods are increased with a slow trend. In other words, the impact of GPS errors is not very obvious for localization accuracy. Besides, SLMLU still has smaller average location error and location error standard deviation than other three schemes.

\subsection{Impact of Radio Range}

Figure 6 describes the variation of the average location error and location error standard deviation as the radio range for the four methods. Note that with the increase of the radio range, the average location errors and location error standard deviation of the four methods are increased.

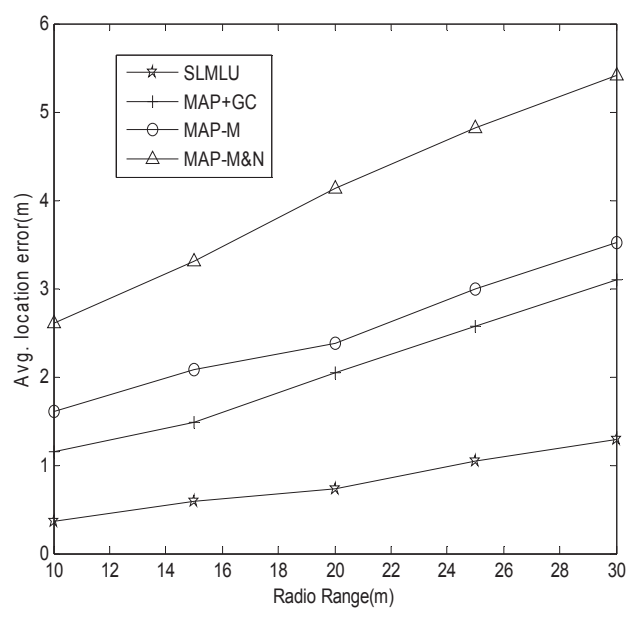

(a)

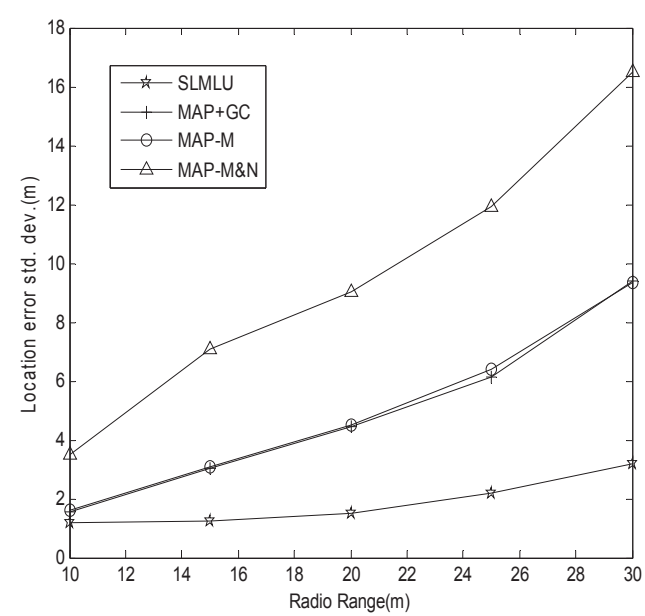

(b)

Fig. 6. Relationship between localization performance and radio range. (a) Average location error. (b) Location error standard deviation. 
A large radio range will lead to a weaker restriction, and ultimately lead to larger average location errors and location error standard deviations of the four schemes. Nevertheless, the impact of radio range on $\mathrm{MAP}+\mathrm{GC}, \mathrm{MAP}-\mathrm{M}$, MAP-M\&N schemes is much greater than SLMLU scheme.

\subsection{Impact of Radio Irregularity}

As the radio range of node is irregular in practical environment, the influence of degree of irregularity (DOI) is discussed in the section. DOI is defined as the maximum radio range variation per unit degree change in the direction of radio propagation as

$$
K_{i}=\left\{\begin{array}{lc}
1, & i=0 \\
K_{i-1} \pm \text { Rand } \times \text { DOI }, & 0<i<360
\end{array}\right.
$$

where $\left|K_{0}-K_{359}\right| \leq$ DOI [39].

Figure 7 compares the average location error and location error standard deviation of these four schemes

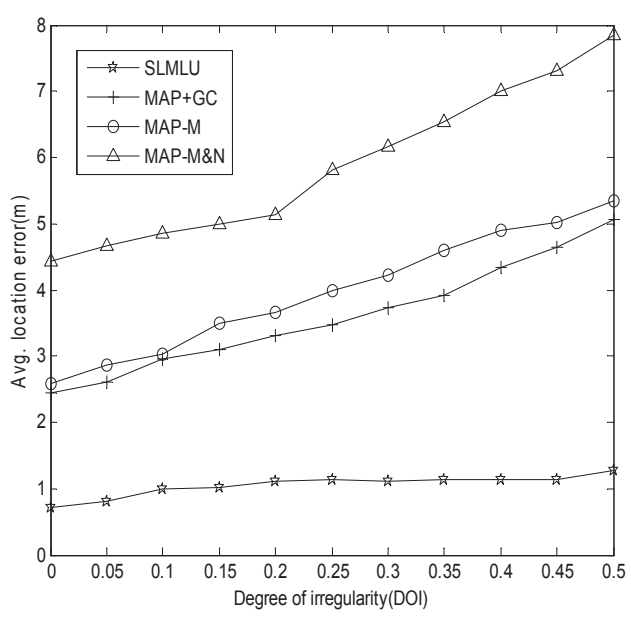

(a)

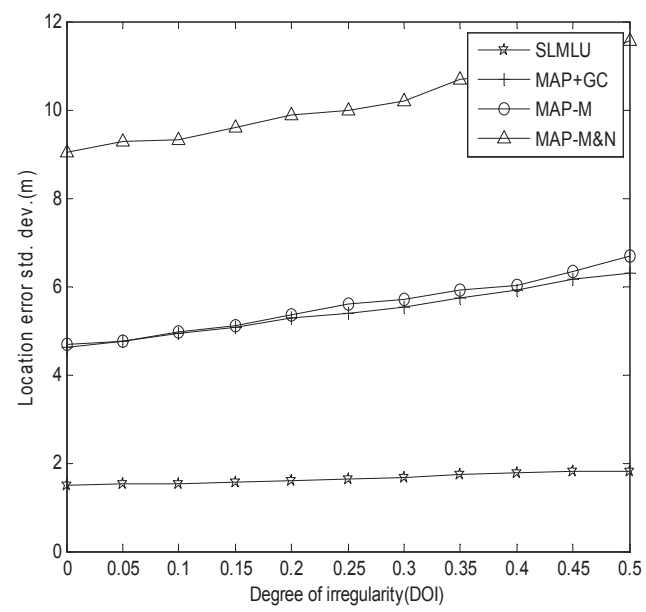

(b)

Fig. 7. Relationship between localization performance and DOI. (a) Average location error. (b) Location error standard deviation. under different DOI. As shown, the average location errors and location error standard deviations of $\mathrm{MAP}+\mathrm{GC}$, MAP-M, MAP-M\&N schemes are increased with the increase of DOI, and DOI has an evident influence on these three schemes, but a slight influence on SLMLU proposed. As MAP+GC, MAP-M, MAP-M\&N schemes make use of the radio range, which is irregular, to estimate the location of the node, DOI has great effect on them. However, SLMLU use RSS to compute the location of the node, so DOI has little effect. Therefore, SLMLU has more localization performance.

\subsection{Impact of Noise}

Figure 8 describes the localization performance of SLMLU scheme under different signal-to-noise ratio (SNR). The average location error and location error standard deviation of SLMLU degrades with the decrease of SNR. This is because the estimation of RSS will be more and more inaccurate with the decrease of SNR. From Fig. 8 and Tab. 1, it can be seen that, even in $0 \mathrm{~dB}$ SNR environment, SLMLU scheme still has better localization perform-

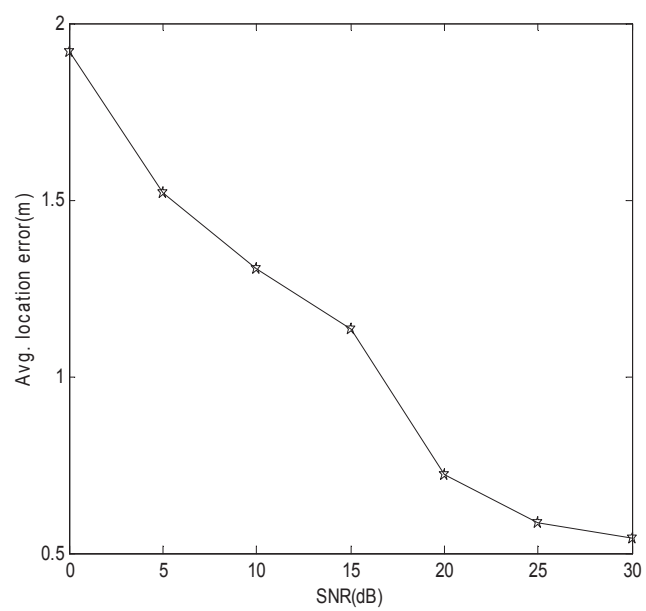

(a)

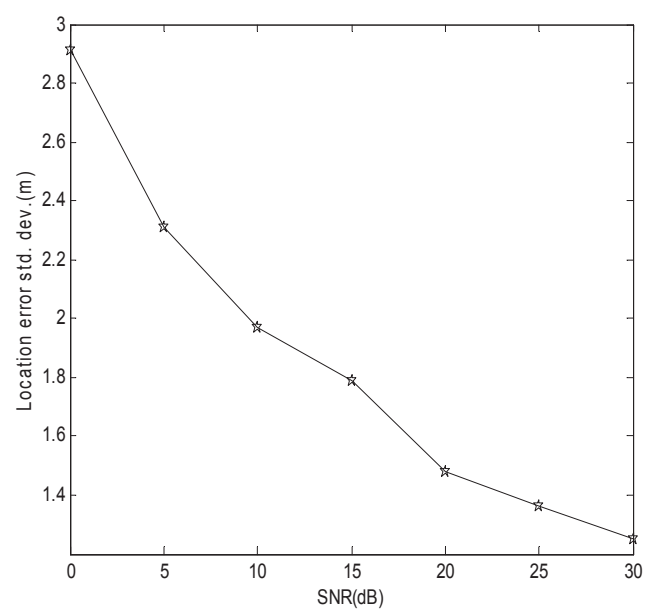

(b)

Fig. 8. Relationship between localization performance and noise. (a) Average location error. (b) Location error standard deviation. 
ance than MAP+GC, MAP-M or MAP-M\&N schemes. That is, SLMLU scheme is still a good choice under extreme low SNR.

\subsection{Complexity Analysis}

In terms of computation cost, each sensor node in $\mathrm{MAP}+\mathrm{GC}$ requires 75 addition/subtraction and 52 multiplication/division operations. Each sensor node in MAP-M and MAP-M\&N our method requires 26 addition/subtraction and 36 multiplication/division operations. The computation complexity of SLMLU is $O(2 M N)$. The computation complexity of SLMLU is more than other three schemes.

\section{Conclusion}

We propose a novel sparse localization algorithm with a mobile beacon based on LU decomposition (SLMLU). First, the scheme makes use of sparse localization model to convert the node localization problem to a 1-sparse vector recovery problem. Then, it adopts LU decomposition pre-processing and compressive sensing (CS) theory to solve the problem of restricted isometry property (RIP) and exactly recover the sparse signal, respectively. Finally, weighted centroid scheme is adopted to exactly locate the node. The simulation results and analysis show that the SLMLU scheme proposed has better localization performance than the previous schemes. As SLMLU adopts the received signal strength (RSS) to localize each node, the obstacles and degree of irregularity (DOI) have little effect on SLMLU, and even under low Signal Noise Ratio (SNR), SLMLU also has better localization performance than $\mathrm{MAP}+\mathrm{GC}, \mathrm{MAP}-\mathrm{M}$, and MAPM\&N schemes. Therefore, SLMLU is more suitable for practical application. In addition, to localize all nodes, SLMLU scheme has a lower requirement of the mobile beacon compared with the previous schemes. However, SLMLU algorithm has a shortcoming of high computational complexity. Therefore, the future research direction will consider how to reduce the computational complexity.

\section{Acknowledgments}

The research is supported by the National Natural Science Foundation of China (Grant No. 61077079), the Key Program of Heilongjiang Natural Science Foundation (Grant No. ZD201216), Program Excellent Academic Leaders of Harbin (Grant No. RC2013XK009003) and the Fundamental Research Funds for the Central Universities (Grant No. HEUCF1508).

\section{References}

[1] BAHL, P., PADMANABHAN, V. N. RADAR: an in-building RFbased user location and tracking system. In Proceedings of the $19^{\text {th }}$
Annual Joint Conference of IEEE Computer and Communications Societies (INFOCOM). Tel Aviv (Israel), 2000, p. 775-784. DOI: 10.1109/INFCOM.2000.832252

[2] BERGAMO, P., MAZZINI, G. Localization in sensor networks with fading and mobility. In Proceedings of the 13th IEEE International Symposium on Personal, Indoor and Mobile Radio Communications (PIMRC). Lisboa (Portugal), 2002, p. 750-754. DOI: 10.1109/PIMRC.2002.1047322

[3] PRIYANTHA, N. B., CHAKRABORTY, A., BALAKRISHNAN, H. The cricket location-support system. In Proceedings of $A C M$ International Conference on Mobile Computing Networking (MOBICOM). Boston (USA), 2000, p. 32-43. DOI: $10.1145 / 345910.345917$

[4] NICUlESCU, D., NATH, B. Ad Hoc Positioning System (APS) using AoA. In Proceedings of the 22nd Annual Joint Conference of IEEE Computer and Communication Societies (INFOCOM). San Francisco (USA), 2003, p. 1734-1743. DOI: 10.1109/ INFCOM.2003.1209196

[5] NASIPURI, A., LI, K. A directionality based location discovery scheme for wireless sensor networks. In Proceedings of ACM International Workshop on Wireless Sensor Networks Applications (WSNA). Atlanta (Georgia, USA), 2002, p. 105-111. DOI: $10.1145 / 570738.570754$

[6] BRIDA, P., MACHAJ, J., BENIKOVSKY, J. Wireless sensor localization using enhanced DV-AoA algorithm. Turkish Journal of Electrical Engineering and Computer Sciences, 2014, vol. 22, no. 3, p. 679-689. DOI: 10.3906/elk-1204-28

[7] BRIDA, P., MATULA, M., DUHA, J. Using proximity technology for localization in wireless sensor networks. Communications, Scientific Letters of the University of Žilina, 2007, vol. 9, no. 4, p. 50-54.

[8] BULUSU, N., HEIDEMANN, J., ESTRIN, D. GPS-less low cost outdoor localization for very small devices. IEEE Personal Communications, 2000, vol. 7, no. 5, p. 28-34. DOI: $10.1109 / 98.878533$

[9] JUN, W., URRIZA, P., HAN, Y. X., et al. Weighted centroid localization algorithm: Theoretical analysis and distributed implementation. IEEE Transactions on Wireless Communications, 2011, vol. 10, no. 10, p. 3403-3413. DOI: 10.1109/TWC.2011.081611.102209

[10] YANG, X. Y., KONG, Q. R., DAI, X. J. An improved weighted centroid location algorithm. Journal of Xi'An Jiaotong University, 2010, vol. 44 , no. 8, p. 1-4.

[11] NICULESCU, D., NATH, B. DV based positioning in ad hoc networks. Kluwer Journal of Telecommunications Systems, 2003, vol. 22 , no. 1 , p. 267-280.

[12] SAVARESE, C., RABAEY, J., LANGENDOEN, K. Robust positioning algorithms for distributed ad-hoc wireless sensor networks. In Proceedings of USENIX Annual Technical Conference. Monterey (CA, USA), 2002, p. 317-327.

[13] BULUSU, N., BYEHKOVSKIY, V., ESTRIN, D. Sealable ad hoc deployable RF-based localization. In Proceedings of Grace Hopper Celebration of Women in Computing Conference. Vancouver (Canada), 2002.

[14] TRAN, D. A., NGUYEN, T. Localization in wireless sensor networks based on support vector machines. IEEE Transactions on Parallel and Distributed Systems, 2008, vol. 19, no. 7, p. 981-994. DOI: 10.1109/TPDS. 2007.70800

[15] SICHITIU, M. L., RAMADURAI, V. Localization of wireless sensor networks with a mobile beacon. In Proceedings of IEEE International Conference on Mobile Ad-Hoc Sensor Systems. Fort Lauderdale (FL, USA), 2004, p. 174-183. DOI: 10.1109/MAHSS. 2004.1392104 
[16] SUN, G. L., GUO, W. Comparison of distributed localization algorithms for sensor network with a mobile beacon. In Proceedings of IEEE International Conference on Networking, Sensing and Control (ICNSC). Taipei (Taiwan), 2004, vol. 1, p. 536-540. DOI: 10.1109/ICNSC.2004.1297496

[17] XIA, Z., CHEN, C. A localization scheme with mobile beacon for wireless sensor networks. In Proceedings of International Conference ITS Telecommunications. Chengdu (China), 2006, p. 1017-1020. DOI: 10.1109/ITST.2006.288725

[18] XIAO, B., CHEN, H. K., ZHOU, S. G. Distributed localization using a moving beacon in wireless sensor networks. IEEE Transactions on Parallel and Distributed Systems, 2008, vol. 19, no. 5, p. 587-560. DOI: 10.1109/TPDS.2007.70773

[19] OU, C. H. A localization scheme for wireless sensor networks using mobile anchors with directional antennas. IEEE Sensors Journal, 2011, vol. 11, no. 7, p. 1607-1616. DOI: 10.1109/JSEN.2010.2102748

[20] SSU, K. F., OU, C. H., JIAU, H. C. Localization with mobile anchor points in wireless sensor networks. IEEE Transactions on Vehicular Technology, 2005, vol. 54, no. 3, p. 1187-1197. DOI: 10.1109/TVT.2005.844642.

[21] LEE, S., KIM, E., KIM, C., et al. Localization with a mobile beacon based on geometric constraints in wireless sensor networks. IEEE Transactions on Wireless Communications, 2009, vol. 8, no. 12 , p. 5801-5805. DOI: 10.1109/ISSNIP.2007.4496820.

[22] LIAO, W. H., LEE, Y. C., KEDIA, S. P. Mobile anchor positioning for wireless sensor networks. IET Communications, 2011, vol. 5, no. 7, p. 914-921. DOI: 10.1049/iet-com.2010.0336

[23] CANDS, E. Compressive sampling. In International Congress of Mathematicians, 2006, vol. 3, p. 1433-1452

[24] CANDS, E., WAKIN, M. An introduction to compressive sampling. IEEE Signal Processing Magazine, 2008, vol. 25, no. 2, p. 21-30. DOI: 10.1109/ MSP.2007.914731

[25] FENG, C., VALAEE, S., TAN, Z. H. Multiple target localization using compressive sensing. In Proceedings of the 28th IEEE Conference on Global Telecommunications. Honolulu (USA), 2009, p. 4356-4361. DOI: 10.1109/GLOCOM.2009.5425808

[26] ZHAO, C. H., XU, Y. L. Energy constraint Bayesian compressive sensing detection algorithm. Journal on Communications, 2012, vol. 33 , no. $10, \quad$ p. 1-6. DOI: 10.3969/j.issn.1000436X.2012.10.001

[27] ZHANG, B., CHENG, X., ZHANG, N., et al. Sparse target counting and localization in sensor networks based on compressive sensing. In Proceedings of the IEEE International Conference on Computer and Communications Societies (INFOCOM). Shanghai (China), 2011, p. 2255-2263. DOI: 10.1109/INFCOM.2011. 5935041

[28] CHEN, S. S., DONOHO, D. L., SAUnders, M. A. Atomic decomposition by basis pursuit. Society for Industrial and Applied Mathematics, 2001, vol. 43, no. 1, p. 129-159. DOI: $10.1137 / \mathrm{S} 003614450037906 \mathrm{X}$

[29] MALLAT, S. G., ZHANG, Z. F. Matching pursuit with timefrequency dictionaries. IEEE Transactions on Signal Processing, 1993, vol. 41, no. 12, p. 3397-3415. DOI: 10.1109/78.258082

[30] TROPP, J. A., GILBERT, A. C. Signal recovery from random measurements via orthogonal matching pursuit. IEEE Transactions on Information Theory, 2007, vol. 53, no. 12, p. 4655-4666. DOI: 10.1109/TIT.2007.909108

[31] CHENG, L., WU, C., ZHANG, Y., et al. A survey of localization in wireless sensor network. International Journal of Distributed Sensor Networks, 2012, p. 1-12. DOI: 10.1155/2012/962523
[32] STOJMENOVIC, I. Handbook of Sensor Networks: Algorithms and Architectures. New York: Wiley, 2005.

[33] BU, C. J., LOU, Y. S. Matrix Theory. Harbin: Harbin Engineering University Press, 2008, p. 83-85.

[34] ALINE, B., KOEN, L. Monte-Carlo localization for mobile wireless sensor networks. Ad Hoc Networks, 2006, vol. 6, no. 5, p. 718-733. DOI: 10.1016/j.adhoc. 2007.06.004

[35] BROCH, J., MALTZ, D. A., JOHNSON, D. B., et al A performance comparison of multi-hop wireless ad hoc network routing protocols. In Proceedings of ACM International Conference on Mobile Computing and Networking (MobiCom). Dallas (TX, USA), 1998, p. 85-97. DOI: 10.1145/288235.288256

[36] IEEE Standard online resource provided by IEEE 802.15 WPAN. [Online]. Available: http://www. ieee802.org/15/pub/TG4.html.

[37] JARDOSH, A., BELDING-ROYER, E. M., ALMEROTH, K. C., et al. Towards realistic mobility models for mobile ad hoc networks. In Proceedings of ACM International Conference on Mobile Computing and Networking (MobiCom 2003). San Diego (CA, USA), 2003, p. 217-229. DOI: 10. 1145/938985. 939008

[38] Geodetic Survey Division-Geodesy-GPS Accuracy Levels. [Online]. Available: http://www. geod.nrcan. gc.ca /edu/geod/gps/gps13_e.php.

[39] HE, T., HUANG, C., BLUM, B. M., et al. Range-free localization schemes for large scale sensor network. ACM Transactions on Embedded Computing Systems, 2005, vol. 4, no. 4, p. 877-906. DOI: $10.1145 / 938985.938995$

\section{About the Authors ...}

Chunhui ZHAO was born in Heilongiang, China, in 1965. He received his B. S. and M. S. degree from Harbin Engineering University, in 1986 and 1989, respectively. He received his Ph.D degree from Harbin Institute of Technology in 1998. He was a postdoctoral research fellow in the College of Underwater Acoustical Engineering of Harbin Engineering University. At present, he is working in the College of Information and Communication Engineering of Harbin Engineering University as a professor and doctoral supervisor. He is a senior member of Chinese Electronics Academy. He has published four works and more than 400 papers. His research interests include digital signal and image processing, communication signal processing, nonlinear filters.

Yunlong XU received his B.E. degree in Communication Engineering from Information Engineering School at Nanchang University in June 2009. He is currently pursuing the Ph.D. degree in Signal and Information Processing at Harbin Engineering University. His research interests include compressive sensing and sensor network.

Hui HUANG received her B.E. degree in Communication Engineering from College of Information and Communication at Harbin Engineering University in June 2009. She is currently pursuing the Ph.D. degree in Communication and Information System at Harbin Engineering University. Her research interests include cognitive radio and localization. 